Research Article

\title{
Deteriorated and Dislocated Multiple Organizational Relationships: An Investigation of Chinese Rock Burst Prone Coal Mines
}

\author{
Shuai Han $\mathbb{D}^{1},{ }^{1}$ Izhar Mithal Jiskani $\mathbb{D}^{2,3}$ Yue Li ${ }^{2},{ }^{4}$ Zhen Liu, ${ }^{1}$ and Jueli Yin ${ }^{1}$ \\ ${ }^{1}$ College of Economics and Management, Shandong University of Science and Technology, Qingdao, China \\ ${ }^{2}$ State Key Laboratory of Coal Resources and Safe Mining, China University of Mining and Technology, Xuzhou, China \\ ${ }^{3}$ School of Mines, China University of Mining and Technology, Xuzhou, China \\ ${ }^{4}$ Coal Economic Research Institute, Shandong Technology and Business University, Yantai, China
}

Correspondence should be addressed to Shuai Han; hessa1222@163.com and Izhar Mithal Jiskani; imjiskani@hotmail.com

Received 5 July 2021; Revised 2 August 2021; Accepted 25 August 2021; Published 8 September 2021

Academic Editor: Jun Wang

Copyright (c) 2021 Shuai Han et al. This is an open access article distributed under the Creative Commons Attribution License, which permits unrestricted use, distribution, and reproduction in any medium, provided the original work is properly cited.

\begin{abstract}
This study explores the multiple organizational relationships between frontline miners, managers, and supervisors to reveal the human organizational risks of coal mine safety and health management. Data were collected from six high-risk rock burst underground mining companies operating in western, central, north-eastern, and south-eastern regions of China. A total of 1105 respondents from the three core groups were investigated. Descriptive statistics and paired test methods were used to empirically analyze the deteriorated and dislocated relationships between multiple roles. The specific conclusions are as follows: (1) Miners' perception of relationship quality is the lowest, and the managers' perception of relationship quality is the highest. (2) "Closeness" relationship is expressed among peer colleagues for all multiple roles. (3) The deteriorated relation rate of miners averagely reached $19.67 \%$, and that of supervisors averagely reached $17.63 \%$, thereby mostly reaching $27.8 \%$ for miners with regard to supervisors. (4) The workers in high positions easily have a phenomenon of "overestimated confidence" in the perception of dislocated relationships, and the "miners-supervisors" and "supervisors-manager" dual-core contradiction have obviously been emerging. (5) The valuable, harmonious, and extent degree are relatively lowest in all relationship items.
\end{abstract}

\section{Introduction}

Mine health and safety constitute an integral part of every mining operation and one of the most important aspects of sustainable mining [1]. Rock burst is a major dynamic disaster that directly threatens safety in coal mines. Rock burst prone coal mines with the inevitably increasing mining depth and intensity are hazardous workplaces requiring serious attention to mine safety [2]. Physical factors such as ground fracturing, principal stresses measurements, microelectromechanical systems, and other risk factors have been extensively studied for rock burst prone coal mines [3-5], while human factors have mostly been ignored in this area. Traditional human behavior studies for coal mine safety have concentrated on unsafe behaviors, occupational injuries, and hidden dangers [6, 7]. Literature reveals that the relational quality and priority among various functional roles such as managers, supervisors, and employees are related to safety activities $[6,8]$. The harmonious and high quality of the organizational relationship is the basic requirement for developing a safe and healthy enterprise [9], which is true for the particular field of the coal industry. The butterfly effect of human factors becomes a dangerous threat for accidents in mines. In addition, the current depressed environment for overcapacity reduction of coal, the increased risk of employee unemployment, the reduced sense of social and professional identity, and the weakened profitability of Chinese coal mines resulted in conflicts due to unbalance of the low income, low recognition, and high requirements. Conflicts between managers, supervisors, and 
frontline miners intensify and diffuse the distrust between them. There is no resultant force among multiple roles, which results in serious human risks and hidden dangers for accidents.

However, there is a certain slow lag for multiple organizational relationship research in coal mine safety management. Meanwhile, the exchange of leadership members in the field of organizational relationship research is a hot topic, which provides new thoughts for traditional coal mine multiple role studies. Leader-member exchange (LMX) thoughts break through the single relationship of organizational support and leadership support to two sides and interactive relationship. Additionally, the employee-organization relationship (EOR) and team-member exchange (TMX) both provide interactive views for multiple roles studies. There is no doubt that interactive relationship plays a key role in organizational performance, and high LMX is always characterized by mutual trust, respect, and loyalty. In general, the lower the LMX variance within a team or the higher the team members' LMX, compared to the team's mean LMX, the better the teamwork results [10]. A healthy and harmonious organizational relationship can undoubtedly improve work performance and output. Organizational factors have been emphasized by many scholars in coal mine safety and health management $[11,12]$. However, the studies on the multiple roles' interactive relationship with each other in the coal mine system are still missing. Therefore, this study examines the relationship between multiple organizational members (frontline miners, managers, and supervisors) to reveal whether there is a good organizational environment and a misalignment in the quality of the relationship between members with different responsibilities and identities in the rock burst prone coal mine system. This study will provide new research ideas for mine safety and health research.

\section{Literature Review}

2.1. Human Factors in Coal Mine Safety and Health Management. Most of the studies on human factors of coal mine safety and health management are focused on the miners, whose unsafety behavior has always been the main content of the research. Unsafe behaviors include specialized and generalized concepts. The specialized unsafe behaviors specifically refer to habitual behaviors with noncompliance for safety policies and procedures, which are primarily attributed to physiological aspects, cognitive aspects, safety knowledge and awareness, work habits, safety training, safety atmosphere, and other factors [13-15]. The generalized sense of unsafe behaviors covers all behaviors that pose hidden dangers to safe production, such as counterproductive behaviors [16], burnout behaviors [17, 18], quit behavior, and ignorance behavior [19]. The generalized unsafe behavior expands the paradigm of attribution factors for a safety issue and changes from the coal mine unsafe behavior paradigm to the healthy behavior paradigm. The internal influencing factors gradually evolve to the perspectives of miners' intrinsic safety perception commitment, moral restraint, and mental health [20-22]. However, the limitations were appearing based on the single role of coal miners since individuals do not exist alone. These studies are breaking away from the behavioral research paradigm in the context of a multirole environment. Meanwhile, some scholars have begun to pay attention to the important role of organizational elements and multirole interaction. Some scholars believe that there is a positive relationship between coal mine employees' safety awareness and organizational culture. Karen stated that the size and diversity of organizations have related effects on coal mine safety accidents [11]. Through literature review, it is found that organizationrelated factors are usually one of the comprehensive elements for safety behavior, mainly focusing on organizational culture and organizational systems, lacking independence and systematic studies for multiple organizational relationships. The fact is that coal mine safety is an important and inseparable output task undertaken by multiple output entities. It is a public output that relies on the "results of the safety behaviors of all occupational roles participated in production actions" [19]. Therefore, the organizational relationship of multiple roles should not be ignored but taken as the core contents for the safety and healthy development of coal mines.

At present, managers and miners have always been the central focus of attention. The research of organizational relationships usually adopts game methods. Yang et al. examined the impact of managers' emotions on miners' behavior using a bilateral signaling game driven by emotion and judgment [23]. Yu et al. described the asymmetry of the game interests between managers and miners with the system dynamics method [24]. It is precisely because this asymmetry affects the stability of workers' behavior, resulting in unsafe behavior. At present, the relationship of multiple roles among managers, frontline miners, supervisors in the coal mine system is relatively weak. Previous studies based on field surveys have shown that miners have a low psychological perception of affections and resource interactions among colleagues, supervisors, and managers. This indicates that the sub-health state was released from the aforementioned roles. Since the availability of high-quality shallow coal has decreased and mining depth and intensity have increased, rock burst disasters have become a significant concern for safe and efficient mining. Prior research has mostly focused on the influence of physical factors, dynamic models, and development and application of rock burst prevention equipment [25-29]. However, the role of unsafe behavior in the occurrence of rock burst accidents has not yet been studied [30]. Therefore, analyzing the relationship quality of multiple roles is essential in order to go over the latent relational risk of safety and health management in coal mines prone to rock bursts.

\subsection{Theoretical Literature Review on Organizational Relationships}

2.2.1. Analysis of the Theoretical Basis of Organizational Relations. The employment relationship is an indispensable content in the study of organizational relations, focused on 
the employer and the employee. The employment relationship is based on the "social exchange theory," which supposed that exchange is the basis of the bilateral relationship, which includes internal and emotional exchange and external and economic exchange [31, 32]. In later period, incentives-contribution theory, organizational support, psychological contract theory, and resource conservation theory emerged based on the social exchange theory, which, respectively, focused on four aspects of individual needs: organizational commitments, responsibilities, obligations of both parties, and resource supply and demand [33, 34]. These basic theories provided the basis for the existence of the quality of the employment relationship. As a whole, there has been a variety of vertical and parallel research works on internal organizational relationships, such as employee-organization relationship (EOR), leadermember exchange (LXM), and team-member exchange (TMX) research. EOR reflects the status of the relationship between employees and their organization in internal management [35]. The quality of EOR is not only related to the vital interests of employees but also important to organizational task performance, organizational citizenship behavior, etc. [36, 37]. However, in the actual organization and management, agent-leaders as actual roles performed the interactive and coordinated activities between the organization and employees. More scholars have begun to use the leader to replace the organization as the direct role since the organization is a virtual and unclear concept for the employee. After that, the binary relation between leaders and members has gradually been of great concern. A good quality relationship between leaders and employees is always characterized by high trust, responsiveness, mutual influence, and support [38]. Meanwhile, a high-quality interaction between leaders and members reduces employees' sense of insecurity at work, improves work performance and enthusiasm, and improves work and family $[9,39]$. However, the current studies still focus on one-way relationships measured from the employee's perspective while ignoring the dual relationship between the leader and the employee in the organization. Although the concepts of "mean" and "difference" were adopted in the LMX studies, they pointed out the interactive differences in the relationship between leaders and members. However, the studies of relational measurement still were conducted from the signal perspective of employees [40, 41]. In addition to the employeeorganization or the employee-leader vertical relationship, the horizontal team relationship is also particularly important in the organizational relationship. Based on the exchange theory framework, team-member exchange is the interaction of views and help between team members, thereby forming a mutually beneficial relationship [42]. This kind of reciprocal performance among team members is also an important part of multiple relationships. Individuals with high-quality team-member exchanges are more willing to work hard for the team and concurrently receive rewards such as acceptance, trust, and support from the team. On the other hand, individuals with low-quality team-member exchanges lack cooperation, trust, and social returns with team members [43]. It could be seen that the differentiated exchange features of multiple roles are attracting more scholars, whether it is the exchange of leadership members or team members. Martin et al. distinguished between two different LMX methods, namely, "shared perspective of the team" and "unique perspective of the follower," to predict the different outcomes in terms of the individual level and team level [10]. Although these measurements are based on the perceptive of employees, differentiated relational cognition has appeared in the study of interaction among leaders or team members. To sum up, it can be seen that the current literature does not address multiple organizational roles or the differences of their interaction as a potential cause of risks in the coal mine sector. EOR, LMX, and TMX offered multiple ideas about the organizational relationship between different roles and proved the quality of role relationship directly related to work output. The related studies will provide theoretical support for multiple entities' relationships in the coal mine safety and health management.

\subsubsection{Attributes of the Relationship Quality of Organization} Members. The most representative EOR scale in the organizational field was designed by Tasui et al. [36]. It included two dimensions, with a total of 7 items: (1) expected contribution ( 3 items) refers to measuring the degree of human resource practices that encourage employees to focus on job performance by the employer; (2) the incentives provided (4 questions) are measured by the degree of training and employment safety provided to employees by the employer. In addition to the scale developed by Tasui et al., the EOR scale further developed by Hon et al. is also used by some scholars. The scale draws on the definition of public relations and subdivides EOR quality into six parts: satisfaction, trust, commitment, mutual control, exchange relationship, and mutual relationship, with a total of 30 questions.

The most representative scale for LMX was developed by Gerstner [44] with a total of 7 items. Through meta-analysis, it is found that the reliability coefficient is relatively high, between 0.8 and 0.9 [44]. The scale included the following items: "manager could understand your problems and needs in work" and "manager would use his power to help me out of trouble". This scale is evaluated by employees. In the TMX study, another common scale developed by Seers to measure team-member exchange is commonly used, with 10 items in total, such as "in a busy situation, team colleagues will ask me to help" and "willing to help me complete the tasks that were assigned to me by the leader" [42].

In addition to the above-mentioned classic relationship scales, many scholars have also put forward their insights on relationship cognition. For example, Namisango and Kang measured the level of relationship between the organization and the public from three aspects: relationship strength, cohesion, and symmetry [45]. As mentioned earlier, the research originated in Western countries and adapted to the individualism in Western culture and the characteristics of social relations regulated by contract and exchange. However, the interdependence and ethics of social relations are emphasized in traditional Chinese culture. The kind of 
affection was strengthened in a Chinese context. The relationship carries different attributes, such as extent and density, depth, and intimacy $[35,46]$.

\subsection{Organizational Relationship Framework among Multiple} Roles in Coal Mines. The contribution of safety outputs from the specific functional divisions of labor is considered. Frontline miners, managers, supervisors, and auxiliary staff are divided into four groups: the executive group, the decision group, the supervision group, and the supportive group. The first three groups account for $80 \%$ to $90 \%$ of the total manpower and become the core entities involved in the coal mine safety production. The decision group mainly refers to the leadership group (management body) responsible for production and operation decision-making and command functions of the coal mine enterprise. It includes the chief of the mine, engineers in charge of professional departments, directors, technical personnel, and deputy directors. Frontline leaders include district captains, technicians, and workshop (deputy) directors. The supervisors have the duties of safety supervision and hazard elimination, mainly charged by the safety supervision department. They also provide support for the safe production process by finding the hazards from the environment or humans and undertaking safety education and training for miners. The executive group has the duties of the special task under the mine, working in the team groups. They have the duties of frontline work in different departments, such as driving, ventilation, transport, waterproofing, and electricity. The reports of previous coal mine accidents reveal that the leading causes of the accidents came from the unsafe behaviors of miners, especially facing the rock burst. The support group is the auxiliary function for the production and operation of the enterprise, such as the staff in the financial department and labor union.

To sum up, according to the contribution of safety output, miners, managers, and supervisors are particularly important in the entire system. This study takes these three roles as the main research objects for subsequent consideration. The relational quality among the three roles is particularly relevant not only to their organizational stability and work performance but also to the sustainable development of safety and health in coal mines. Thus, this study would explore their multiorganizational relationship, aiming to address two major questions: (1) What is the relationship quality perceived by each role with regard to the other roles, i.e., miners, managers, supervisors? (2) Are there dislocated relations between multiple roles, e.g., miners and managers, miners and supervisors, managers and supervisors?

\section{Research Design and Survey}

3.1. Variable Measurement. A questionnaire was designed based on the literature [44] and revised according to the context of Chinese culture. The questionnaire included basic information about the workers and multirole organizational relationship scales. After modifying based on reliability and validity analyses, the final questionnaire consisted of 14 selfreported items. The individual information has seven items: participants' age, work experience, education, number of people/children supported, marital status, religion, and (family's) monthly income. The remaining items were about the organizational relationship scale, which consisted of 7 items to measure the multiple work roles' relationship perceptions with each role based on the organizational relationship scale. The multiple work role relationship includes frontline miners' relationship, managers' relationship, and supervisors' relationship. The items included are shown in Table 1. The questionnaire was designed based on the 10point Likert scale, ranging from 1 "highly disagree" to 10 "highly agree." Questionnaire items were used for both groups of workers except for differences in targeted roles.

3.2. Sample. Deep mining has resulted in an increase in coal mine accidents, mainly caused by rock bursts. The later accident analysis shows that human factors are a significant contributor to these accidents. For that reason, this study was conducted at six coal mines that are at high risks of rock bursts. These mining companies are located in western, central, north-eastern, and south-eastern regions of China. The research team visited mine sites to distribute questionnaires and collect the data. Participants were male Chinese coal frontline miners, managers, and supervisors.

The research team encountered many difficulties throughout the whole survey process. First, arrangements were done for a suitable time to fill questionnaires, taking into account the avoidance of conflicts between participants' working hours and time to complete the questionnaire. Participants completed their questionnaires individually prior to their work shift. Second, to ensure accurate filling, management personnel were requested to leave the site during the process of completing the questionnaire. Third, preparations were made to ensure that participants understand each of the questionnaire items. Since some aged and less educated workers have low literacy, one-to-one help was provided to them to improve their understanding and help them choose their appropriate response.

As part of the presurvey, a total of 350 questionnaires were distributed. Of those, 305 valid questionnaires were collected from participants in an anonymous manner. The reliability and validity of the questionnaire were analyzed based on the item analysis and principal component analysis using SPSS software. The results showed that the questionnaire has good reliability and validity. In the formal survey phase, a total of 1310 questionnaires were distributed. With an effective recovery rate of $85 \%$, the number of participants for this study reached 1115 . Of all participants, 661 were miners, 244 were managers, and 200 were supervisors. Their partial demographic details are given in Table 2.

Frontline miners aged below 30 accounted for 15.3\%, a percentage higher than that of managers and supervisors. Managers and supervisors over the age of 40 are significantly more than miners, showing that miners are younger than others. In terms of income per month, the managers' income per month above $8000 \mathrm{RMB}$ accounted for $35.7 \%$. However, 
TABLE 1: Scale of organizational relationship.

\begin{tabular}{|c|c|c|}
\hline Scale items & Contents & Scale $(1-10)$ \\
\hline $\begin{array}{l}\text { Harmonious } \\
\text { degree }\end{array}$ & $\begin{array}{c}\text { ou have a mutually beneficial connection that is balan } \\
\text { miners }\end{array}$ & $\begin{array}{l}\text { Highly disagree- } \mathrm{h} \\
\text { agree }\end{array}$ \\
\hline Extent degree & There's some intersection of life and work between managers/supervisors/miners and you & $\begin{array}{r}\text { Highly dis } \\
\text { ag }\end{array}$ \\
\hline Density degree & You have enough interactions and communications with your ma & $\begin{array}{r}\text { Highly dis } \\
\text { as }\end{array}$ \\
\hline Dept & Man & $\begin{array}{r}\text { Highly dis } \\
\text { a }\end{array}$ \\
\hline Intimacy degree & anagers/supervisors $/ \mathrm{m}$ & Highly dis \\
\hline Valu & Managers & $\begin{array}{l}\text { Highly disagree-highly } \\
\text { agree }\end{array}$ \\
\hline Thicl & $\begin{array}{l}\text { You have enough confidence in managers/super } \\
\text { defend and explain the decisions they } \mathrm{m}\end{array}$ & $\begin{array}{l}\text { Highly disagree-highly } \\
\text { agree }\end{array}$ \\
\hline
\end{tabular}

TABLE 2: The percentage of demographic variables of all samples.

\begin{tabular}{|c|c|c|c|c|}
\hline Demographic variables & Categories & Frontline miners (\%) & Managers (\%) & Supervisors (\%) \\
\hline \multirow{6}{*}{ Age } & $<30$ & 15.3 & 7.0 & 5.0 \\
\hline & $31-35$ & 16.5 & 17.2 & 16.0 \\
\hline & $36-40$ & 15.6 & 20.5 & 17.5 \\
\hline & $41-45$ & 23.6 & 19.3 & 30.0 \\
\hline & $46-50$ & 19.2 & 23.4 & 18.0 \\
\hline & $>51$ & 9.8 & 12.7 & 13.5 \\
\hline \multirow{6}{*}{ Monthly family income (RMB) } & $\leq 2000$ & 9.4 & 2.0 & 7.0 \\
\hline & $2000-4000$ & 41.3 & 30.7 & 54.0 \\
\hline & $4000-6000$ & 29.8 & 23.4 & 31.5 \\
\hline & $6000-8000$ & 15.9 & 8.2 & 6.5 \\
\hline & $8000-10,000$ & 2.7 & 11.5 & 0.0 \\
\hline & $>10,000$ & 0.9 & 24.2 & 1 \\
\hline \multirow{5}{*}{ Number of supported people } & 1 people & 5.0 & 3.3 & 3.0 \\
\hline & 2 people & 8.8 & 10.7 & 11.0 \\
\hline & 3 people & 29.8 & 36.9 & 38.5 \\
\hline & 4 people & 31.2 & 33.2 & 24.0 \\
\hline & $>4$ people & 25.0 & 16.0 & 23.5 \\
\hline \multirow{7}{*}{ Education } & $<\mathrm{PS}$ & 1.1 & 0.8 & 1.5 \\
\hline & PS & 3.9 & 1.6 & 1.5 \\
\hline & JS & 41.8 & 13.9 & 30.5 \\
\hline & HS; ST & 23.1 & 12.7 & 27.0 \\
\hline & JC & 17.5 & 16.0 & 18.0 \\
\hline & $\mathrm{U}$ & 10.6 & 46.3 & 21.0 \\
\hline & $\mathrm{P}$ & 2.0 & 8.6 & 0.5 \\
\hline
\end{tabular}

Note. Education includes the following: P (postgraduate); U (undergraduate); JC (junior college); ST (secondary technical); HS (high school); JS (junior school); PS (primary school).

only $3.6 \%$ of miners and $1 \%$ of supervisors reach above 8000 RMB. Supervisors' income per month between 4000 and 6000 reached $86.5 \%$, which is lower than that of miners. In terms of education, more than half of managers have at least undergraduate degree $(46.3 \%$ undergraduate and $8.6 \%$ postgraduate), but more than half of miners and supervisors have below high school education (frontline miners: $41.8 \%$ junior school, 23.1\% high school; supervisors: $30.5 \%$ junior school, $27.0 \%$ high school). The number of supported people is nearly close among the three roles; the proportion supporting around 3 or 4 people are the most, and managers supporting people above 5 are relatively less than miners and supervisors.
3.3. Reliability and Validity Analysis. SPSS 22.0 and AMOS 21.0 as common software were used to test the reliability and validity of the data. The reliability of the questionnaire was analyzed in terms of Cronbach's $\alpha$ and item-to-total analysis (Table 3). Multiple subjects are abbreviated in all tables and figures. F stands for frontline miner, $\mathrm{S}$ represents supervisor, $\mathrm{M}$ represents manager, and $\mathrm{R}$ represents relationship. Accordingly, the F-MR denotes frontline miners' perception of their relationship with managers.

The questionnaire has good reliability as the Cronbach's $\alpha$ values of all factors are between 0.888 and 0.936 , and those for the survey of frontline miners, managers, and supervisors are $0.938,0.942$, and 0.955 , respectively. The item-to-total 
TABLE 3: Reliability and validity analysis results of organizational relationship scale.

\begin{tabular}{|c|c|c|c|c|}
\hline Scales & Role & Classification & Cronbach's $\alpha$ & Item-to-total \\
\hline \multirow{9}{*}{ Organizational relationship of multiple roles } & \multirow{3}{*}{ Frontline miners $(\mathrm{F})$} & F-MR & 0.900 & $0.525-0.851$ \\
\hline & & F-SR & 0.891 & $0.589-0.783$ \\
\hline & & F-WR & 0.914 & $0.509-0.833$ \\
\hline & \multirow{3}{*}{ Managers (M) } & M-FR & 0.890 & $0.590-0.752$ \\
\hline & & M-SR & 0.888 & $0.529-0.747$ \\
\hline & & M-WR & 0.911 & $0.612-0.812$ \\
\hline & \multirow{3}{*}{ Supervisor (S) } & S-MR & 0.911 & $0.566-0.807$ \\
\hline & & S-FR & 0.916 & $0.681-0.804$ \\
\hline & & S-SR & 0.936 & $0.659-0.827$ \\
\hline
\end{tabular}

coefficient ranged between 0.509 and 0.851 , proving a good validity based on the principle that the item-to-total coefficient was higher than 0.3 .

Additionally, a further validity test was conducted using AMOS. After adjustments for the mode of organizational relationship, the fitting indexes reached a better range. The indicators of organizational relationship were CMIN/DF: 4.288, GFI: 0.987, RMSEA: 0.071, NFI: 0.992, AGFI: 0.948, RFI: 0.976, IFI: 0.994, TLI: 0.981, and CFI: 0.994. These results prove good reliability and validity of the multirole organizational relationship scale. Moreover, principal component analysis was performed to export factors structure for survey items. The KMO values of frontline miners, managers, and supervisors are 0.923, 0.898, and 0.909 , respectively. Bartlett's test score for sphericity was significant $(p<0.001)$. The three variables in the frontline miners' questionnaire reached $72.853 \%$ of the variance. The F-MR, F-SR, F-WR values were 29.931\%, 52.802\%, and $72.853 \%$, respectively. In the manager's questionnaire, the variance in FR, M-SR, and M-WR was recorded as $46.830 \%$, $58.489 \%$, and $66.006 \%$, respectively. The findings of the supervisor questionnaire showed that S-MR, S-FR, and S-WR were $53.143 \%, 63.500 \%$, and $70.714 \%$, respectively.

\section{Data Analysis}

4.1. The Average Analysis of the Relational Quality of Multiple Roles. The whole relational quality of multiple roles by the average analysis method is directly presented. Every role has three relational connections with others. In every connection, three types were identified: deteriorated relation $[1,4]$, medium relation $(4,7]$, and closeness relation $(7$, 10]. Deteriorated relation (DR) represents the poor quality of the relationship. Medium relation (MR) represents the general pass quality of the relationship. Closeness relation (CR) represents the high and closeness quality of the relationship.

It can be seen from the average quality of the relationship between miners, managers, and supervisors in Table 4 that the relationship quality of miners is the lowest (5.831), the relationship quality of managers is the highest (6.456), and the relationship quality of supervisors is in the middle (5.996). As a whole, the relationship with regard to peer colleagues is the highest compared with other different roles for miners, managers, and supervisors. The relationship quality of miners regarding managers and supervisors has not exceeded the intermediate level (5.5), showing a poor relational situation. Through the analysis of deteriorated, medium, and closeness relations, it can be found that the average deteriorated relation rate of miners and supervisors, respectively, reached $19.67 \%$ and $17.63 \%$. The deteriorated relation rate of miners with regard to managers and supervisors is relatively high $(23.75 \%, 27.38 \%)$, while that of managers with regard to other roles is relatively low $(8.61 \%$, $11.07 \%)$, and deteriorated relations of supervisors with regard to managers is relatively high $(27.36 \%)$. The results show that the relationship quality among multiple roles is not optimistic.

4.2. Analysis of Relationship Quality of the Same Role. The same role has a different perception for other roles' relationship; hence, the spatial distribution, difference analysis, and specific items of the same role's relationship quality with other multiple roles should be further explored to reveal the hidden relationship characteristics.

\subsubsection{Scatter Diagram of Relationship Perception of the Same} Roles with Regard to Other Multiple Roles. The position of each point represents the relationship quality of frontline miners with the manager, supervisor, and colleagues in Figure 1(a). It can be seen that certain differences in relationship quality were presented. The most points were distributed in the score ranges between 4 and 6. Significantly, the concentration of low scores is apparent for miners with regard to managers and supervisors. At the same time, on the MS mapping surface, the dots are nearly in a straight line, indicating that the miners have a consistent effect on the relationship cognition between managers and supervisors. In Figure 1(b) for managers, the points are mainly concentrated between 6 and 7. Compared with miners, the distribution of points is slightly shifting to the space of high value, indicating that managers are relatively optimistic about the perception of the relationship with multiple roles. In Figure 1(c) for supervisors, the points are mostly concentrated between and 5 and 7. Compared with managers, there are more singularities (asymptotic boundary points) distributed. For each mapping surface of MS $\backslash S C \backslash M C$, all projection is a relatively straight line compared to the other two roles, indicating that the supervisor's cognitive consistent effect is more substantial. 
TABLE 4: Average analysis result of multirole relational quality.

\begin{tabular}{|c|c|c|c|c|c|c|}
\hline Role & Classification & Mean value & Standard deviation & Deteriorated relation rate $[1,4]$ & $\begin{array}{c}\text { Medium relation rate } \\
(4,7](\%)\end{array}$ & $\begin{array}{c}\text { Closeness relation rate } \\
(7,10](\%)\end{array}$ \\
\hline \multirow{4}{*}{ F (\%) } & F-MR & 5.564 & 2.12 & 23.75 & 47.35 & 28.90 \\
\hline & F-SR & 5.299 & 2.217 & 27.38 & 51.59 & 21.03 \\
\hline & F-FR & 6.630 & 1.942 & 7.87 & 51.59 & 40.54 \\
\hline & Mean value & 5.831 & 2.093 & 19.67 & 50.18 & 30.16 \\
\hline \multirow{4}{*}{ M } & M-FR & 6.44 & 1.726 & 8.61 & 57.38 & 34.02 \\
\hline & M-SR & 6.197 & 1.776 & 11.07 & 57.79 & 31.15 \\
\hline & M-MR & 6.730 & 1.687 & 6.15 & 48.77 & 45.08 \\
\hline & Mean value & 6.456 & 1.730 & 8.61 & 54.65 & 36.75 \\
\hline \multirow{4}{*}{ S } & S-MR & 5.448 & 2.197 & 27.50 & 51.50 & 21.00 \\
\hline & S-FR & 6.241 & 2.042 & 12.12 & 56.57 & 31.31 \\
\hline & S-SR & 6.301 & 1.925 & 13.27 & 55.10 & 31.63 \\
\hline & Mean value & 5.996 & 2.055 & 17.63 & 54.39 & 27.98 \\
\hline
\end{tabular}

Note. F: frontline miners, M: managers, S: supervisors.

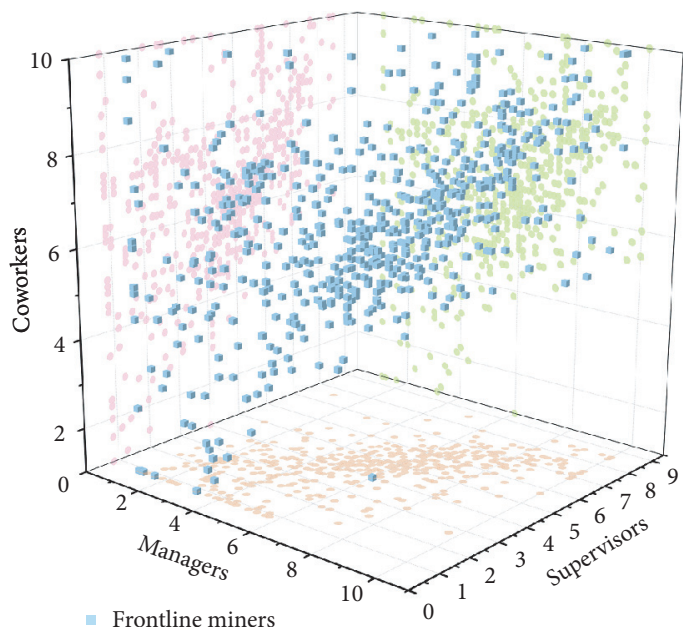

(a)

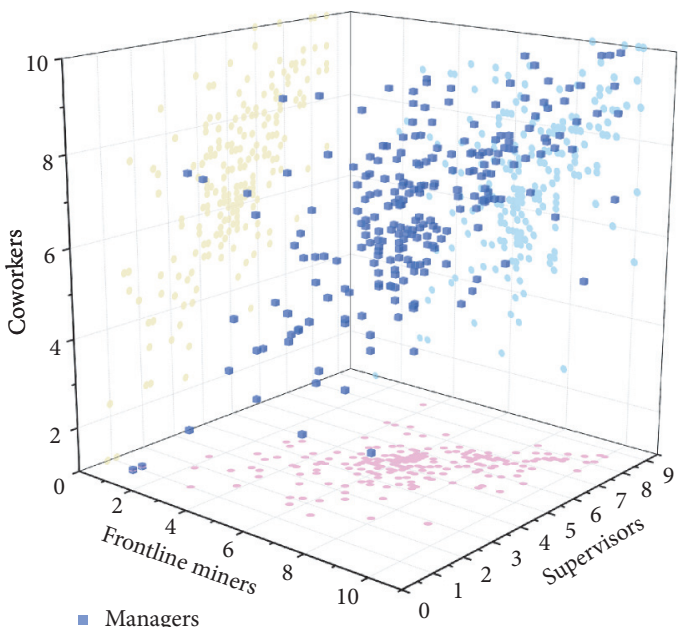

(b)

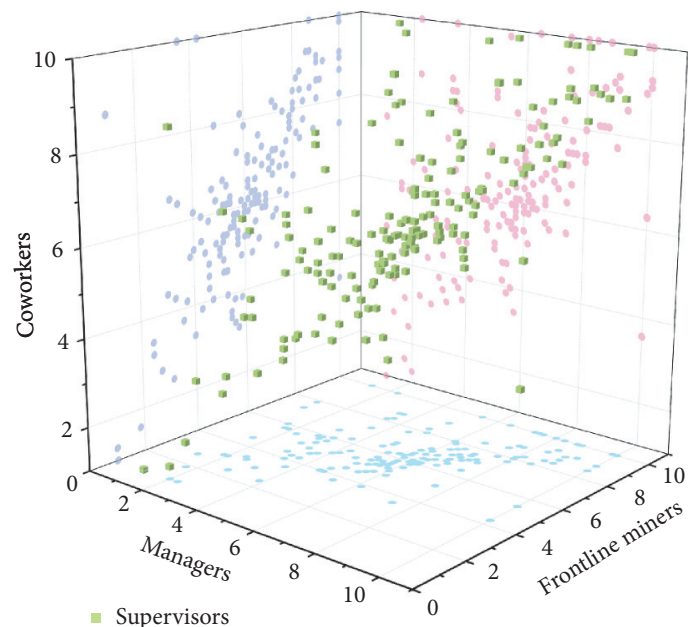

(c)

Figure 1: Scatter diagram of relationship perception of (a) frontline miners, (b) managers, and (c) supervisors.

4.2.2. Difference Analysis of Relationship Quality of the Same Role with Regard to Other Multiple Roles. It is found that the relationship quality perceptions of multiple groups presented certain degrees of dispersion and differences based on the scatter diagram. Hence, it is necessary to conduct a different analysis based on the method of the paired sample $t$-test. 
The results show significant differences in the relationship perception of frontline miners regarding managers, supervisors, and coworkers (Table 5). Among them, the relationship quality with managers is higher than that with supervisors (mean differences $=0.957$, sig. $=0.000$ ). The relationship quality with coworkers is higher than that with supervisors and managers (mean differences $=-2.023$, mean differences $=-1.065$, sig. $=0.000)$.

As for the managers, there are significant differences in the relationship perception with regard to frontline miners, supervisors, and coworkers. Among them, the relationship quality with miners is higher than that with supervisors (mean differences $=1.101$, sig. $=0.000$ ). The relationship quality with coworkers is higher than that with supervisors and frontline miners (mean differences $=-1.391$, mean differences $=-0.290$, sig. $=0.000)$.

As for the supervisors, there are significant differences in the relationship perception with regard to frontline miners, managers, and coworkers. Among them, the relationship quality with miners is higher than that with managers (mean differences $=1.101$, sig. $=0.000$ ), and the relationship quality with coworkers is higher than that with managers (mean differences $=-0.794$, sig. $=0.000)$. There are no significant differences among coworkers and frontline miners.

4.2.3. Subitem Analysis of Relationship Perception of the Same Roles with Other Multiple Roles. The subitem analysis is used to reveal the specific and different performances for interactive relationships; multiple roles show different distribution in the seven degrees of relationship perception. The subitems analysis of frontline miners, managers, and supervisors is as follows.

Figure 2(a) shows that the overall average of harmonious and extent degree of miners are relatively lowest, and every subitem with coworkers is higher than other roles. The relationship quality of miners with coworkers is relatively highest among all roles. In terms of coworkers, the highest value is intimacy degree (mean $=7.041$ ), and the lowest is the valuable degree (mean $=5.992)$, followed by managers with the highest value being depth degree $($ mean $=6.115)$ and the lowest being extent degree $($ mean $=4.905)$. The relationship quality with supervisors is overall worst. Among them, harmonious degree (mean $=4.844)$, extent degree $($ mean $=4.752)$, and density degree $($ mean $=4.828)$ are all lower than 5 points, indicating that these degrees of relationship quality are quite poor.

Figure 2(b) shows that the average of the overall items of managers is relatively higher and closer than the radar chart of frontline miners. The relationship quality of managers with coworkers is relatively highest compared to other roles in terms of all items. In particular, the highest value is thickness degree (mean=7.393), and the lowest is the valuable degree $($ mean $=6.516)$. This is followed by frontline miners, with the highest value being depth degree (mean$=6.823$ ) and the lowest being valuable degree (mean$=6.041$ ). The relationship quality for supervisors is overall worst. Among them, extent degree (mean=5.988) and density degree $($ mean $=5.816)$ are both lower than 6 points, indicating that the relationship quality is relatively poor.
Figure 2(c) shows that the relationship quality of supervisors with managers is obviously lower than that with frontline miners and coworkers. In terms of coworkers, the highest value is thickness degree $($ mean $=6.750)$, and the lowest is a harmonious degree (mean $=5.955)$. This is followed by frontline miners, with the highest value being thickness degree $($ mean $=6.845)$ and the lowest being the harmonious degree $($ mean $=5.945)$. The last is the relationship quality with managers; the value of the overall subitem was lower than 6 points, and the harmonious degree was the lowest $($ mean $=4.945)$.

4.3. Dislocation Analysis of Different Roles' Relationship Quality. The different analysis for the same role has been explored in previous section. However, interactive analyses among different roles should be further explored, such as the bilateral analysis and dislocation levels analysis of different roles.

It can be found that there is a phenomenon of "dislocation" between the different bilateral roles in Figure 3. The quality perception of managers is higher than that of miners in both bilateral relationships. The same is true in terms of the bilateral relationship between managers and supervisors; the quality perception of managers is higher than that of supervisors. In terms of the bilateral relationship between miners and supervisors, the quality perception of supervisors is higher than that of miners. To sum up, miners are relatively pessimistic in the perception of heterogeneous role relationships. In contrast, managers' perception of interpersonal relationships is excessively optimistic. This study divides the dislocation status into five levels based on the function of the ratio of (the low of role A-the high of role B)/ role $\mathrm{A}$ ). When the misalignment exceeds $50 \%$, this means that the relationship scale has been completely out of balance. Therefore, the dislocation is classified between $0 \%$ and $50 \%$, respectively: deviated dislocation $(0 \sim 10 \%, \mathrm{I})$, distant dislocation [10 20\%, II), exclusionary dislocation [20 30\%, III), obstructive dislocation [30 40\%, IV), and fractured dislocation [40 50\%, V) (see Table 6).

It can be seen that the overall dislocation ratio is between 9.7\% and $32 \%$ in Table 5. Among them, the dislocations of frontline miners and managers, and supervisors and managers are relatively severe, showing the exclusionary dislocation. This is followed by supervisor and managers, and miners and managers, showing the distant dislocation. Regarding the specific subitems of the relationship perception, the overall differences in the extent degree, harmonious degree, and density degree are obviously larger.

\section{Discussion}

The poor organizational relationship leads to burnout of coal mine employees. It is detrimental to the retention of loyal and responsible employees in coal enterprises and reduces employees' safety participation $[47,48]$. The results of data analysis revealed that miners, managers, and supervisors have deteriorated and dislocated relationships with each other, which brings great human risks to sustainable mine 
TABLE 5: Paired test analysis of the same role with other multiple roles.

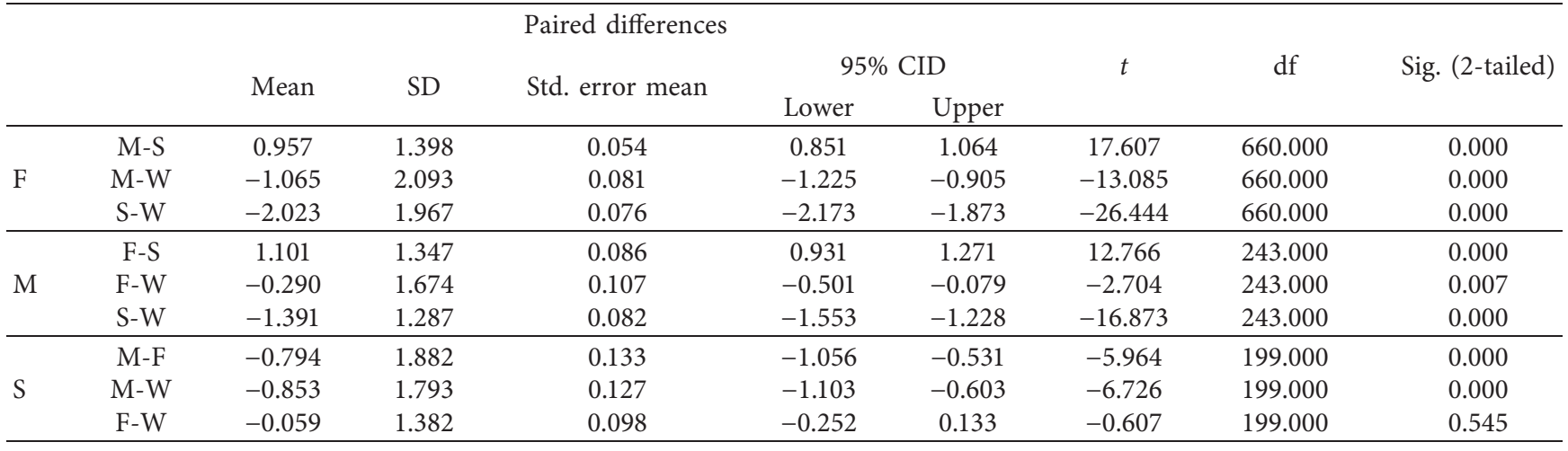

Note. SD: standard deviation; CID: confidence interval of the difference.

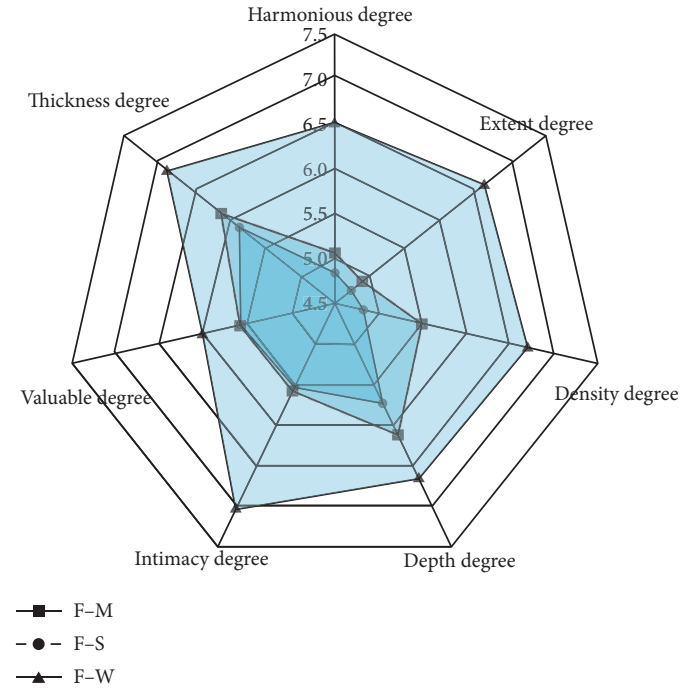

(a)

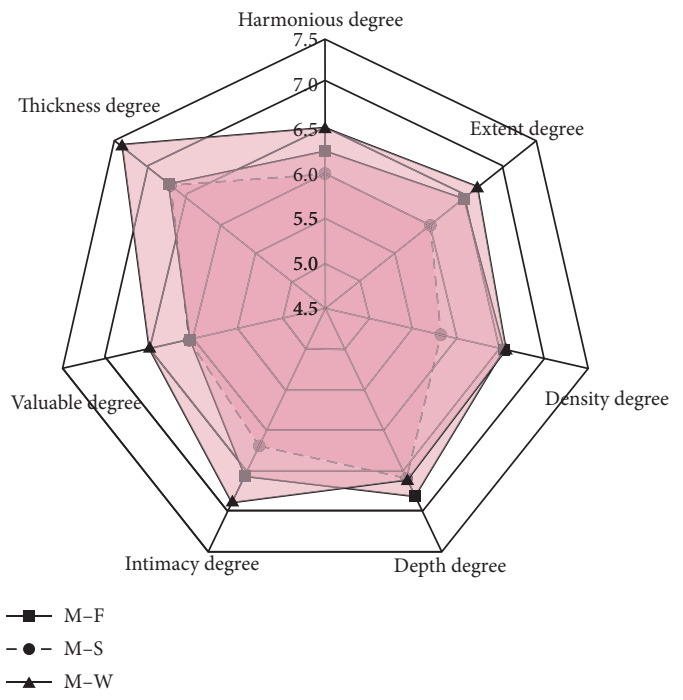

(b)

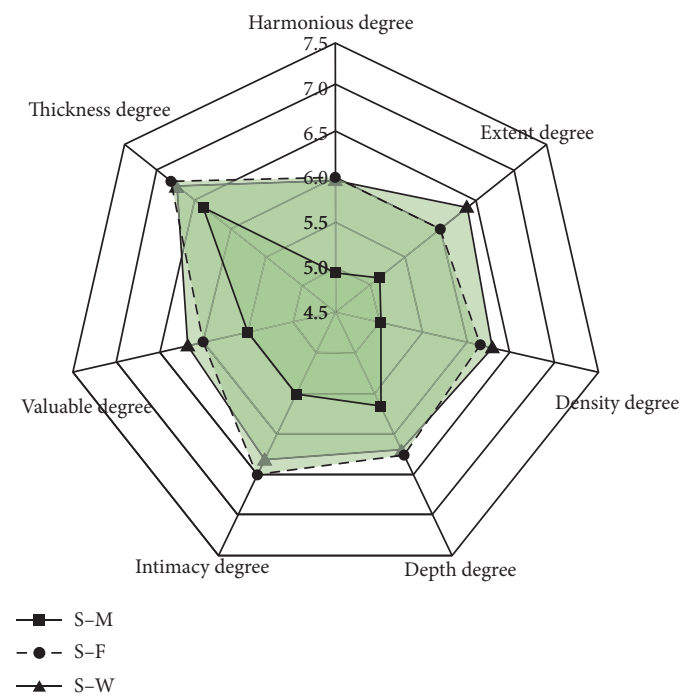

(c)

FIGURE 2: A subitems radar chart of the same role's relationship perception with other multiple roles: (a) frontline miners, (b) managers, (c) supervisors. 


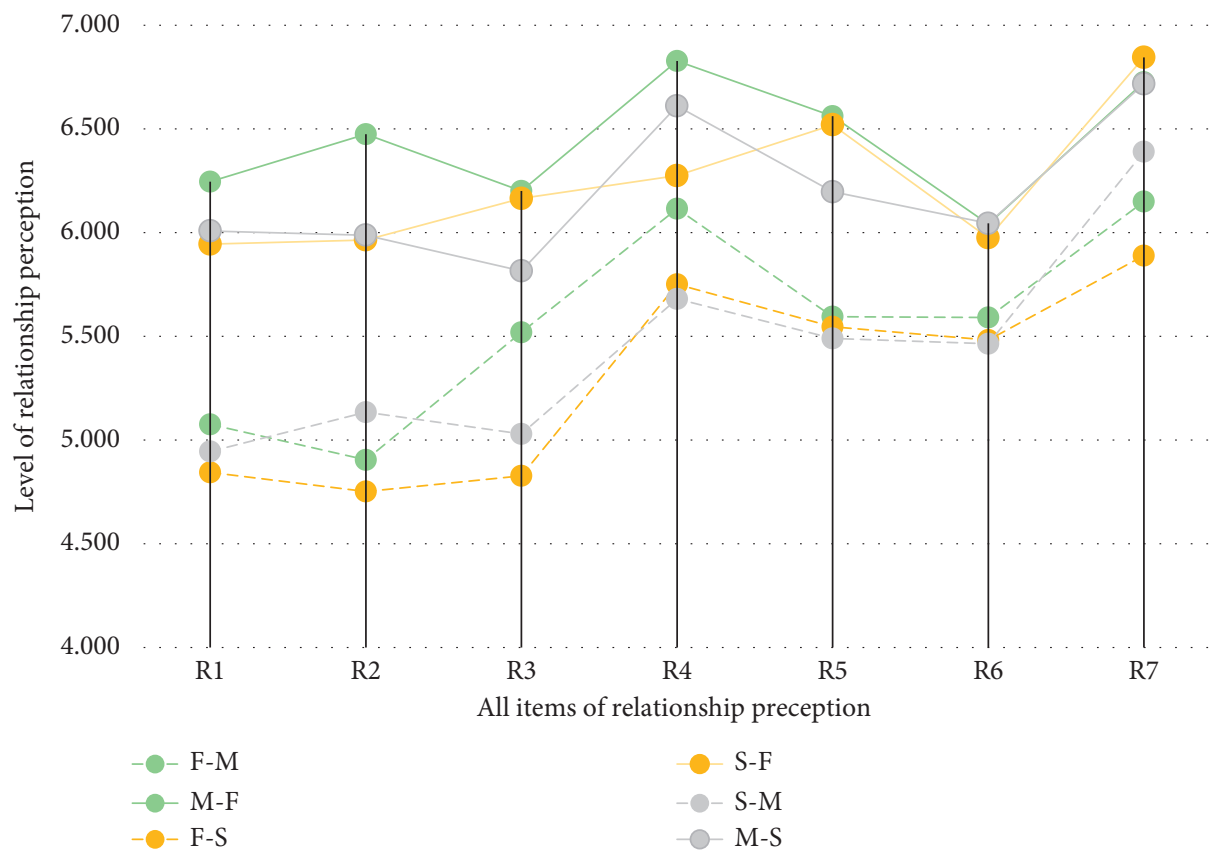

FIGURE 3: Bilateral analysis of different roles' relationship quality. Note. R1 R6 represent, respectively, harmonious degree, extent degree, density degree, depth degree, intimacy degree, valuable degree, and thickness degree.

TABLE 6: Dislocation levels analysis of different roles' relationship quality.

\begin{tabular}{lccccccccc}
\hline & $\begin{array}{c}\text { Harmonious } \\
\text { degree (\%) }\end{array}$ & $\begin{array}{c}\text { Extent } \\
\text { degree } \\
(\%)\end{array}$ & $\begin{array}{c}\text { Density } \\
\text { degree (\%) }\end{array}$ & $\begin{array}{c}\text { Depth } \\
\text { degree } \\
(\%)\end{array}$ & $\begin{array}{c}\text { Intimacy } \\
\text { degree (\%) }\end{array}$ & $\begin{array}{c}\text { Valuable } \\
\text { degree (\%) }\end{array}$ & $\begin{array}{c}\text { Thickness } \\
\text { degree (\%) }\end{array}$ & $\begin{array}{c}\text { Mean } \\
\text { Dislocation } \\
\text { levels }\end{array}$ \\
\hline F\&M & -23.1 & -32.0 & -12.4 & -11.7 & -17.3 & -8.0 & -9.4 & -15.83 & II \\
F\&S & -22.7 & -25.5 & -27.7 & -9.1 & -17.6 & -9.0 & -16.2 & -17.78 & II \\
S\&M & -21.5 & -16.6 & -15.6 & -16.4 & -12.9 & -10.6 & -5.1 & -18.89 & II \\
Mean & -22.4 & -24.7 & -18.6 & -12.4 & -15.9 & -9.2 & -10.2 & -13.75 & II \\
$\begin{array}{l}\text { Dislocation } \\
\text { levels }\end{array}$ & III & III & II & II & II & I & II & II & II \\
\hline
\end{tabular}

safety and healthy development. Miners have the lowest perception of the relationship quality of other groups, while managers' perception of the relationship quality of other groups is the highest. In addition, compared with other colleagues (i.e., miners, managers, and supervisors), the relationship quality between colleagues at the same level is high.

According to the average value of each dimension of relationship quality, the miners have the weakest relationship perception, and the managers have the strongest relationship perception. Compared with other groups, all roles' relationship with coworkers is relatively high. This result confirms our previous view that there exist conflicting emotional and resource interactions among the miners, managers, and supervisors. It is also shown that a poor symbiosis was released from interpersonal interactions between the organizational roles in the coal mine system. Similarly, it has been found that all roles have a close relationship with their colleagues, which is consistent with the result of Han et al. [6]. The reason why the quality of relationships between coworkers is high is that they have more opportunities to get familiar with and support each other in their work and life. In terms of scatter diagram analysis of multirole relationship perception, there was an obvious phenomenon for miners; that is, the concentration of low scores for relationship quality was obvious with managers and supervisors. Afterward, the significant existence of these differences was verified by the paired sample $t$-test method. Miners have the lowest relationship with supervisors, followed by managers and coworkers. Batt [49] and $\mathrm{Lu}$ and $\mathrm{Li}$ [50] indicated that currents rules increased the conflicts between workers and supervisors, such as check targets and penalties for violations. Additionally, the organizational constraints reflect a particular work environment, which inhibited the individual performance of work tasks [51]. As a disadvantaged group, miners have long been subjected to hierarchical gradient culture, unfairness, and hierarchical barriers. Their loyalty and sense of responsibility have been destroyed. Thus they indirectly disregard the operational rules [7, 52-54]. Meanwhile, they are responsible for executing frontline work and mastering the details and difficulties of underground work. Their work was always often overloaded. However, they do not have enough bargaining power to assert their rights due to many reasons such as the 
migrants' identity, low qualifications, and fewer feedback channels. Often, the policies issued by managers can only be implemented mechanically, and sometimes the policies were outdated [1]. In addition, the internal reasons of employees also affect the relationship. Robert and Vandenberghe [55] proposed the concepts of internal work control locus, which means a sense of control over the work environment. They indicated that workers' influence of internal work control locus could primarily impact their contribution and loyalty in organizational interaction. Internal work control locus also works for LMX since it was as one kind of organizational interaction performance. Based on this, frontline miners seriously lack the internal work locus of control since many coal miners are still relocated and were sacked in the present de-capacity period of coal.

Previous studies primarily focused on miners but ignored the managers and supervisors, leading to a lack of studies on the quality of bilateral multirole relationships [40]. In fact, managers' or supervisors' perceptions could change the miners' perception to some extent. Schopf et al. pointed out a significant negative association between the trustworthiness of supervisors and employees' safety behaviors [56]. For the managers, the relationship perception for colleagues is the highest, and the relationship perception for miners is higher than that of the supervisors. Managers often show the image of a positive status and commander in the work management. They tend to be concerned about things from a long-term optimistic view, which easily leads to an overperception of their relationship with other roles. For the supervisors, the relationship perception for miners is higher than that of the managers. These results indicate an exciting phenomenon; that is, the relationship between supervisors and miners was always focused on. However, the relationship between supervisors and managers is worse. There are fewer studies that prove this, but the reason could be that managers operate the contradiction between supervisors and miners through unreasonable regulatory indicators and rules, in addition to the absence of invalid feedback and enough trust, which was found in the field survey process.

The dislocation data shows that dual-core characteristics of the main contradiction exactly existed in coal mine organizations, that is, "miners-supervisors" and "supervisors-manager" dislocation contradiction. More studies verified the contradiction between the miners and supervisors $[19,48]$, ignoring the latter contradiction. It also was found that there are larger dislocations in terms of the extent degree, harmonious degree, and density degree. These arguments indirectly reflect on the studies of a harmonious workplace or the social network of employees. Few studies fully discuss this in terms of mine management $[57,58]$. Song and Meier found that the self-evaluations of managers do not often match the evaluation results from employees, which seriously affects the job satisfaction of employees [59]. Interestingly, this phenomenon is actually happening in coal mining enterprises. The people in high positions are overconfident about the perception of relationships. For example, managers are positive side, and miners/supervisors are negative side in their relationship; supervisors are positive side, and miners are negative side in their relationship, which provides a new direction for improving the cognition of the actual work environment.

In addition, it was found that the overall average of valuable, harmonious, and extent degree is relatively lowest. This indirectly proves that communication or contact frequency is not enough either in work or in life, and a mutually beneficial relationship is not formed. The valuable degree is relatively weak for colleagues, and harmonious and extent degree are relatively weak for other roles. The manager's perception of value is relatively weak compared to the other two roles, which indicated that other roles' value contribution is weakened. Although there are few directly related studies for various relational degrees, they provide a directional guide for improving relationship quality in the future.

\section{Conclusion}

By investigating the relationship quality of the frontline miners, supervisors, and managers in coal mine organizations, this study discovered that the average relationship quality of each entity is between 5.831 and 6.456 , which is not optimistic. At the same time, the study revealed that the deteriorated and dislocated relationships between multiple roles were obvious. The main findings are as follows:

(i) Multiple roles have the highest relationship quality with their coworkers. The average value of the relationship quality is between 6.301 and 6.730 .

(ii) There are noticeable differences between the same role and other multiple roles in the deteriorated relationship. These differences are manifested in terms of average, proportion of three levels of deteriorated relationship, and scattered points.

(1) First of all, from the mean value of relationship quality, it can be known that the relationship perception of the miners is the highest, and the relationship perception of the managers is the lowest. The significant existence of these differences is verified by the paired sample $t$-test method. For miners, the relationship quality order was presented as follows: supervisors $<$ managers $<$ workers. For managers, the relationship quality order was presented as follows: supervisors $<$ miners $<$ coworkers. For supervisors, the relationship quality order was presented as follows: managers $<$ miners $<$ coworkers.

(2) The proportions of dislocated relationships for miners and supervisors are relatively high. The dislocated relation rate of miners averagely reaches $19.67 \%$, and that of supervisors averagely reaches $17.63 \%$. Specifically, the dislocated relation rate of miners with regard to managers and supervisors, respectively, reached $23.75 \%$ and $27.38 \%$.

(3) The points of the multirole relationship perception were differently distributed in the three-dimensional map. Significantly, the concentration of low scores 
for frontline miners is apparent for relationship quality with managers and supervisors.

(4) The people in high positions easily have a phenomenon of overestimated confidence in the perception of relationships. In addition, the "minerssupervisors" and "supervisors-manager" dual-core contradiction have obviously been emerging.

(5) Overall, the particle items of relationship degree, i.e., valuable, harmonious, and extent degree, are relatively lowest. The valuable degree is relatively weak for colleagues, and harmonious and extent degrees are relatively weak for other roles.

The above results examine the hidden human risks from the organizational micro perspective in the process of sustainable development of coal mine safety and health. This study verified the deteriorated relationship and dislocated relationship in the organizational interaction, which solves the shortcomings of a single perspective in the human studies in coal mine management. The study provides a direction for multirole management for sustainable coal mine development. However, this research also has the following shortcomings: Firstly, this research is a case study based on coal mining enterprises, only representative coal companies from different regions were randomly selected, and it was quite hard to gain more extensive coal data due to the limits of remote regions and economic cost. Secondly, the analysis method is relatively simple. This study reveals the situation of organizational relations and hidden characteristics through the intuitive analysis and difference analysis of the case data. In the future, the relation evolution should be concerned with a dynamic perspective to reveal the relational improvement and safety and health outcomes by solving the deteriorated relationship and dislocated relationship.

\section{Data Availability}

The data used to support the findings of this study are included within the article.

\section{Conflicts of Interest}

The authors declare that they have no conflicts of interest or personal relationships that could have appeared to influence the work reported in this paper.

\section{Authors' Contributions}

Shuai Han contributed substantially to the conception, research design, and analysis. Izhar Mithal Jiskani participated in the methodology and drafting. Yue Li helped in revising the manuscript. Zhen Liu and Jueli Yin assisted in data collection.

\section{Acknowledgments}

This work was financially supported by the National Natural Science Foundation of China (Grant no. 72004123), Natural Science Foundation of Shandong Province (Grant no.
ZR201911180111), Shandong Province Social Science Foundation (Grant no. 21DGLJ17), and Outstanding Youth Innovation Team Project of Universities in Shandong Province.

\section{References}

[1] I. M. Jiskani, S. Han, A. U. Rehman, N. M. Shahani, M. Tariq, and M. A. Brohi, "An integrated entropy weight and grey clustering method-based evaluation to improve safety in mines," Mining, Metallurgy and Exploration, vol. 38, no. 4, pp. 1773-1787, 2021.

[2] P. Makowski and Z. Niedbalski, "A comprehensive geomechanical method for the assessment of rockburst hazards in underground mining," International Journal of Mining Science and Technology, vol. 30, no. 3, pp. 345-355, 2020.

[3] S. Ji, J. Zhang, R. Pan, and J. Karlovšek, "Local acceleration monitoring and its application in physical modelling of underground mining," International Journal of Rock Mechanics and Mining Sciences, vol. 128, no. 6, Article ID 104282, 2020.

[4] R. Gao, T. Kuang, Y. Zhang, W. Zhang, and C. Quan, "Controlling mine pressure by subjecting high-level hard rock strata to ground fracturing," International Journal of Coal Science and Technology, vol. 8, pp. 1-15, 2021.

[5] D. Yang, Z. Ning, Y. Li, Z. Lv, and Y. Qiao, "In situ stress measurement and analysis of the stress accumulation levels in coal mines in the northern Ordos Basin, China," International Journal of Coal Science and Technology, 2021.

[6] S. Han, H. Chen, R. Long, and R. Long, "Who reports low interactive psychology status? an investigation based on Chinese coal miners," International Journal of Environmental Research and Public Health, vol. 17, no. 10, p. 3446, 2020.

[7] I. M. Jiskani, S. Han, N. M. Shahani, M. Ali, I. Dianat, and S. R. Chalgri, "Evaluation of physical and environmental working conditions of underground coal mines within the framework of ergonomics," International Journal of Mining and Mineral Engineering, vol. 11, no. 3, pp. 240-256, 2020.

[8] Y. H. Huang, W. R. Chang, J. H. Cheung, and J. Lee, "The role of employee perceptions of safety priorities on safety outcomes across organizational levels," Ergonomics, vol. 64, pp. 1-30, 2021.

[9] T. M. Probst, L. Jiang, and M. Graso, "Leader-member exchange: moderating the health and safety outcomes of job insecurity," Journal of Safety Research, vol. 56, pp. 47-56, 2016.

[10] R. Martin, G. Thomas, A. Legood, and S. Dello Russo, "Leader-member exchange (LMX) differentiation and work outcomes: conceptual clarification and critical review," Journal of Organizational Behavior, vol. 39, no. 2, pp. 151-168, 2018.

[11] P. Karen, "Blood on the coal: the effect of organizational size and differentiation on coal mine accidents," Journal of Safety Research, vol. 40, no. 2, pp. 85-95, 2010.

[12] L. Jing, Q. Bai, W. Guo, Y. Feng, L. Liu, and Y. Zhang, "Contributory factors interactions model: a new systemsbased accident model," Systems Research and Behavioral Science, vol. 37, no. 2, pp. 255-276, 2020.

[13] H. Yu, H. Chen, and R. Long, "Mental fatigue, cognitive bias and safety paradox in Chinese coal mines," Resources Policy, vol. 52, pp. 165-172, 2017.

[14] L. Fang, Z. Zhang, and H. Guo, "Cognitive mechanism and intervention strategies of coal miners' unsafe behaviors: evidence from China," Revista de Cercetare şi Intervenţie Socială, vol. 61, pp. 7-31, 2018. 
[15] E. J. Haas and P. L. Yorio, "Exploring the differences in safety climate among mining sectors," Mining, Metallurgy and Exploration, vol. 38, no. 1, pp. 655-668, 2021.

[16] B. Marcus and H. Schuler, "Antecedents of counterproductive behavior at work: a general perspective," Journal of Applied Psychology, vol. 89, no. 4, pp. 647-660, 2004.

[17] C. Maslach and M. P. Leiter, The Truth about Burnout: How Organization Cause Personal Stress and What to Do about it, Jossey-Bass, San Francisco, CA, USA, 1997.

[18] S. Clarke, "Contrasting perceptual, attitudinal and dispositional approaches to accident involvement in the workplace," Safety Science, vol. 44, no. 6, pp. 537-550, 2006.

[19] H. Chen, F. Chen, D. Zhu, H. Qi, and R. Long, "Burnout in Chinese coal mine safety supervision," Energy Policy, vol. 85, no. 85, pp. 22-31, 2015.

[20] J. Wei, H. Chen, and H. Qi, "Who reports low safety commitment levels? an investigation based on Chinese coal miners," Safety Science, vol. 80, pp. 178-188, 2015.

[21] H. Lu, H. Chen, W. Du, and R. Long, "Moral values congruence and miners' policy following behavior: the role of supervisor morality," Science and Engineering Ethics, vol. 23, no. 3, pp. 1-23, 2016.

[22] M. Yu and J. Li, "Work-family conflict and mental health among Chinese underground coal miners: the moderating role of psychological capital," Psychology Health and Medicine, vol. 25, no. 1, pp. 1-9, 2020.

[23] X. Yang, Y. Tian, K. Feng, J. Yang, S. Zhang, and S. Wang, "Signal game analysis on the effectiveness of coal mine safety supervision based on the affective events theory," Complexity, vol. 2020, no. 1, 9 pages, Article ID 5710419, 2020.

[24] K. Yu, L. Zhou, Q. Cao, and Z. Li, "Evolutionary game research on symmetry of workers' behavior in coal mine enterprises," Symmetry Plus, vol. 11, no. 2, p. 156, 2019.

[25] K. Zhang, S. Ji, Y. Zhang, J. Zhang, and R. Pan, "MEMS inertial sensor for strata stability monitoring in underground mining: an experimental study," Shock and Vibration, vol. 2018, no. 6, 8 pages, Article ID 4895862, 2018.

[26] D. Z. Song, X. Q. He, E. Y. Wang, and Z. Li, "A dynamic ejection coal burst model for coalmine roadway collapse," International Journal of Mining Science and Technology, vol. 29, no. 4, pp. 36-43, 2019.

[27] X. Liu, D. Fan, Y. Tan et al., "New detecting method on the connecting fractured zone above the coal face and a case study," Rock Mechanics and Rock Engineering, vol. 54, no. 1, pp. 1-13, 2021.

[28] X. Liu, D. Fan, Y. Tan et al., "Failure evolution and instability mechanism of surrounding rock for close-distance parallel chambers with super-large section in deep coal mines," International Journal of Geomechanics, vol. 21, no. 5, Article ID 04021049, 2021.

[29] X. Liu, S. Song, Y. Tan et al., "Similar simulation study on the deformation and failure of surrounding rock of a large section chamber group under dynamic loading," International Journal of Mining Science and Technology, vol. 31, no. 3, pp. 495-505, 2021.

[30] W. T. Yin, "Analysis of rock burst accidents in coal mine based on behavioral safety," Safety In Coal Mines, vol. 52, pp. 245-247, 2021.

[31] K. J. Gergen, Social Exchange: Advances in Theory and Research, Plenum Pub Corp, New York, NY, USA, 1980.

[32] M. S. Mitchell, R. S. Cropanzano, and D. M. Quisenberry, "Social exchange theory, exchange resources, and interpersonal relationships: a modest resolution of theoretical difficulties," Handbook of Social Resource Theory, Springer, Berlin/Heidelberg,Germany, 2012.

[33] J. Pate, "The changing contours of the psychological contract: unpacking context and circumstances of breach," Journal of European Industrial Training, vol. 30, pp. 32-47, 2006.

[34] J. A.-M. Coyle-Shapiro and L. M. Shore, "The employeeorganization relationship: where do we go from here?" $\mathrm{Hu}$ man Resource Management Review, vol. 17, no. 2, pp. 166-179, 2007.

[35] S. L. Zhu, L. R. Long, and W. He, "A study on interest rate channel of monetary policy in China: mediation effect and the difference between inside and outside of the institutional system," Management World, vol. 11, pp. 119-134, 2015.

[36] A. S. Tasui, J. L. Pearce, L. W. Porter, and A. Tripoli, "Alternative approaches to the employee-organization relationship: does investment in employees pay off?" Academy of Management Journal, vol. 40, no. 5, pp. 1089-1121, 1997.

[37] L. Jiwen Song, A. S. Tsui, and K. S. Law, "Unpacking employee responses to organizational exchange mechanisms: the role of social and economic exchange perceptions," Journal of Management, vol. 35, no. 1, pp. 56-93, 2009.

[38] G. Thomas, R. Martin, O. Epitropaki, Y. R. F. Guillaume, and A. Lee, "Social cognition in leader-follower relationships: applying insights from relationship science to understanding relationship-based approaches to leadership," Journal of Organizational Behavior, vol. 34, pp. 63-81, 2013.

[39] V. J. Morganson, D. A. Major, and M. L. Litano, "A multilevel examination of the relationship between leader-member exchange and work-family outcomes," Journal of Business and Psychology, vol. 32, no. 4, pp. 379-393, 2017.

[40] M. J. Martinko, J. D. Mackey, S. E. Moss, P. Harvey, C. P. McAllister, and J. R. Brees, "An exploration of the role of subordinate affect in leader evaluations," Journal of Applied Psychology, vol. 103, no. 7, pp. 738-752, 2018.

[41] D. Zhao, J. Wu, and J. Gu, "Higher-quality leader-member exchange (LMX), higher-level voice? the impact of LMX differentiation and LMX mean on promotive and prohibitive team voice," Current Psychological Research, vol. 4, pp. 1-19, 2020.

[42] A. Seers, "Team-member exchange quality: a new construct for role-making research," Organizational Behavior and Human Decision Processes, vol. 43, no. 1, pp. 118-135, 1989.

[43] L. C. Wang and J. R. Hollenbeck, "LMX in team-based contexts: TMX, authority differentiation, and skill differentiation as boundary conditions for leader reciprocation," Personnel Psychology, vol. 72, no. 2, pp. 271-290, 2018.

[44] C. R. Gerstner and D. V. Day, "Meta-analytic review of leadermember exchange theory: correlates and construct issues," Journal of Applied Psychology, vol. 82, no. 6, pp. 827-844, 1997.

[45] F. Namisango and K. Kang, "Organization-public relationships on social media: the role of relationship strength, cohesion and symmetry," Computers in Human Behavior, vol. 101, pp. 22-29, 2019.

[46] F. L. Liu, G. Z. Yang, and X. F. Geng, Psychology, Harbin Engineering University Press, Harbin, China, 2010.

[47] R. D. Iverson and C. Maguire, “The relationship between job and life satisfaction: evidence from a remote mining community," Human Relations, vol. 53, no. 6, pp. 807-839, 2000.

[48] H. Lu and H. Chen, "Does a people-oriented safety culture strengthen miners' rule-following behavior? the role of mine supplies-miners' needs congruence," Safety Science, vol. 76, no. 76, pp. 121-132, 2015. 
[49] R. Batt, "Who benefits from teams? comparing workers, supervisors, and managers," Industrial Relations, vol. 43, no. 1, pp. 183-212, 2004.

[50] Y. Lu and X. Li, "A study on a new hazard detecting and controlling method: the case of coal mining companies in China," Safety Science, vol. 49, no. 2, pp. 279-285, 2011.

[51] S. Pindek and P. E. Spector, "Organizational constraints: a meta-analysis of a major stressor," Work and Stress, vol. 30, no. 1, pp. 1-19, 2016.

[52] R. Eisenberger, R. Huntington, S. Hutchison, and D. Sowa, "Perceived organizational support," Journal of Applied Psychology, vol. 71, no. 3, pp. 500-507, 1986.

[53] S. Han, H. Chen, R. Long, H. Qi, and X. Cui, "Evaluation of the derivative environment in coal mine safety production systems: case study in China," Journal of Cleaner Production, vol. 143, no. 1, pp. 377-387, 2017.

[54] S. Han, H. Chen, E. Stemn, and J. Owen, "Interactions between organisational roles and environmental hazards: the case of safety in the Chinese coal industry," Resources Policy, vol. 60 , pp. 36-46, 2018.

[55] V. Robert and C. Vandenberghe, "Locus of control and leader-member exchange: a dimensional, contextualized, and prospective analysis," Frontiers in Psychology, vol. 11, pp. 1-14, 2020.

[56] A. K. Schopf, J. Stouten, and W. B. Schaufeli, "The role of leadership in air traffic safety employees' safety behavior," Safety Science, vol. 135, pp. 105-118, 2021.

[57] G. Byun, Y. Dai, Y. Dai, and S. Lee, "A structural view of employee voice: propositions based on the social network perspective," The Korean Academic Association of Business Administration, vol. 29, no. 8, pp. 1241-1259, 2016.

[58] T. Hong, G. Liu, W. W. Ko, and A. Curtis, "Harmonious workplace climate and employee altruistic behavior: from social exchange perspective," International Journal of Manpower, $p$, vol. 42, no. 1, pp. 95-112, 2021.

[59] M. Song and K. J. Meier, "Walking the walk: does perceptual congruence between managers and employees promote employee job satisfaction?" Review of Public Personnel Administration, vol. 2, Article ID 0734371X2096664, 2020. 